\title{
Enhanced harmonic generation in x-ray free-electron lasers
}

\author{
H. P. Freund, ${ }^{*}$ N. A. Yampolsky, and Q. Marksteiner \\ Los Alamos National Laboratory, Los Alamos, New Mexico 87545, USA
}

(Received 19 September 2013; published 21 January 2014)

\begin{abstract}
Harmonic generation becomes increasingly important as $\mathrm{x}$-ray free-electron lasers push to shorter wavelengths. Recent studies have pointed to the possibility of enhancing harmonic generation by detuning the fundamental. In x-ray free-electron lasers, the wiggler line is composed of multiple wiggler segments with magnetic quadrupoles in the gaps to provide for increased focusing. In this paper, we study the effect on harmonic generation in simulation by (1) varying the gap lengths between the wiggler segments and (2) varying the electron beam $\beta$ function. In studying the harmonic we find enhanced harmonic generation is periodic in the gap length and peaks are found as the wiggler separation varies by $\lambda / 3$ (where $\lambda$ is the fundamental wavelength), which corresponds to a phase shift of $2 \pi / 3$. As a consequence, enhanced harmonic generation is found both when the fundamental emission is strong by the nonlinear harmonic generation mechanism and by linear harmonic generation when the fundamental is detuned.
\end{abstract}

DOI: 10.1103/PhysRevSTAB.17.010702

PACS numbers: 41.60.Cr, 52.59.Rz

\section{INTRODUCTION}

Free-electron lasers (FELs) have been designed to operate over virtually the entire electromagnetic spectrum from microwaves through $\mathrm{x}$ rays and in a variety of configurations including amplifiers and oscillators [1]. In recent years, short wavelength, x-ray FELs became operational at SLAC [2] at a wavelength of $1.5 \AA$ and SACLA [3] at a wavelength of $0.634 \AA$, and there is interest building in achieving lasing at still shorter wavelengths. Since the cost of achieving shorter wavelengths by scaling up the electron beam energy is high, there is great interest in operation at harmonics of the fundamental resonance. Nonlinear harmonic generation (NHG) has been studied for some time [4-7], and the expected output power at the $h$ th harmonic is typically of the order of $10^{-h}$ times the power of the fundamental [5]. As a result, various schemes have been proposed to enhance the harmonic interactions [8-11]. In contrast to NHG, linear harmonic generation (LHG) might become important if the fundamental can be suppressed. A method that was proposed for suppressing the fundamental [12] is based upon shifting the phase of the fundamental between wiggler segments. For the third harmonic, this phase shift should be $2 \pi / 3$. Following up on this suggestion, Schneidmiller and Yurkov [13] analyzed the effect of optimizing the $\beta$ function for LHG operation at the third harmonic.

The phase shift between wiggler segments can be controlled by various techniques. For example, a chicane

\footnotetext{
*henryf@lanl.gov

Published by the American Physical Society under the terms of the Creative Commons Attribution 3.0 License. Further distribution of this work must maintain attribution to the author(s) and the published article's title, journal citation, and DOI.
}

can be inserted between the wiggler segments to delay the propagation of the electrons relative to the radiation. This has the advantage that the phase shift can be controlled by the field strength in the chicane. Another approach is simply to vary the gap length between the wiggler segments.

In this paper, we present three-dimensional simulations of the emission at the fundamental and third harmonic as functions of the gap length between wiggler segments and the $\beta$ function. As we will show, the harmonic emission is of the order of $10^{-h}$ as expected for the choice of the optimal $\beta$ function for fundamental radiation. However, as the $\beta$ function increases, both NHG and LHG signatures appear at significantly increased power levels. Indeed, peaks in the third harmonic emission are observed at periodic intervals in the phase shift separated by $2 \pi / 3$ by either the NHG or LHG mechanisms. As a result, detuning of the fundamental is not required to enhance the harmonic generation.

The organization of the paper is as follows. The simulations are conducted using the MEDUSA simulation code, which is briefly described in Sec. II. A general discussion of the phase shift due to varying gap lengths is given in Sec. III. The simulation results for the fundamental and third harmonic as functions of gap length and $\beta$ function are discussed in Sec. IV. Comparisons between the simulation results and analytic models are also discussed. A summary and discussion is given in Sec. V.

\section{THE SIMULATION CODE}

MEDUSA is a three-dimensional simulation code that includes time dependence, harmonics, and start-up from noise [5,14-16]. It models helical and planar wigglers and the optical field is represented as a superposition of GaussHermite modes. Electron trajectories are integrated using 


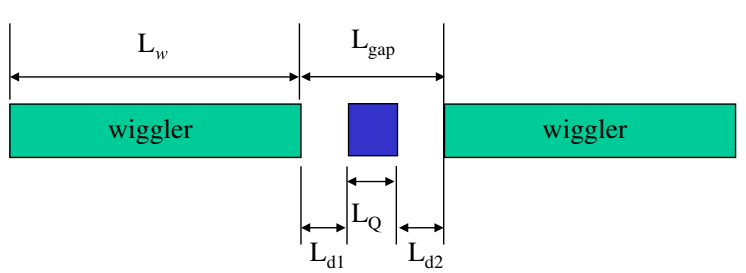

FIG. 1 (color online). Illustration of the placement of the wiggler segments and the quadrupoles in the FODO lattice.

the three-dimensional Lorentz force equations in the combined magnetostatic and optical fields. No wiggler average orbit analysis is used. As such, MEDUSA includes wiggler periods at the entrance and exit from each wiggler segment to describe the transition fields and simulates the injection and ejection of the electrons from each wiggler segment including the phase shift between the electrons and the radiation self-consistently. Models for quadrupoles and dipoles are included. Therefore, MEDUSA provides better confidence in modeling harmonic generation as compared with wiggler-orbit-averaged codes.

MEDUSA has been extensively validated by comparison with amplifier experiments at Brookhaven National Laboratory [17,18], a high average power oscillator at Jefferson Laboratory [19], and the SPARC SASE (selfamplified spontaneous emission) experiment at ENEA Frascati [20].

Here, we simulate an FEL that incorporates a focusing and defocusing (FODO) lattice where the wiggler segments are separated by quadrupoles located in the center of the gaps. This is illustrated in Fig. 1, where the wiggler segments of uniform length $L_{w}$ are separated by a gap of length $L_{\text {gap }}$. The quadrupoles of length $L_{Q}$ are located in the center of the gap with drift spaces $L_{d 1}$ and $L_{d 2}$ of equal length.

\section{INTERWIGGLER PHASE SHIFTS}

We consider the phase shift over the distance from the start of one wiggler segment to the start of the next segment [21]. This includes the phase shift in the wiggler, in the entry and exit tapers and in the gap between the wigglers. The phase shift in the gap is

$$
\varphi_{\mathrm{gap}}=-\frac{L_{\mathrm{gap}}}{\lambda_{w}\left(1+K_{\mathrm{rms}}^{2}\right)},
$$

where $L_{\text {gap }}$ is the separation between the wigglers, $\lambda_{w}$ is the wiggler period, and $K_{\mathrm{rms}}$ is the rms wiggler strength parameter $\left(=0.06602 B_{w} \lambda_{w}\right.$, where $B_{w}$ is the on-axis field strength in $\mathrm{kG}$ and $\lambda_{w}$ is expressed in centimeters). The phase shift in the wiggler is given by one wavelength per wiggler period only when the interaction is on resonance. When the wavelength is detuned from the resonant wavelength $\left(\lambda_{\text {res }}\right)$, then the phase shift in the wiggler is given by

$$
\frac{\varphi_{w}}{2 \pi}=-\frac{L_{w}}{\lambda_{w}}\left(1-\frac{\lambda-\lambda_{\text {res }}}{\lambda_{\text {res }}}\right) .
$$

The phase shift in the entry and exit tapers from the wiggler depends upon the shape of the field variation. We consider a field in the entry region directed in the $y$ direction that increases to a uniform level in one wiggler period as $B_{w, y}(z)=B_{w} \sin ^{2}\left(k_{w} z / 4\right) \cos \left(k_{w} z\right)$, where $k_{w}$ is the wiggler wave number and $z$ is measured from the start of the entry region. Assuming that the exit region is symmetric, then the phase shifts in the two regions are identical and can be expressed as

$$
\frac{\varphi_{\text {trans }}}{2 \pi}=-\frac{2\left(1+3 K_{\mathrm{rms}}^{2} / 8\right)}{\left(1+K_{\mathrm{rms}}^{2}\right)} .
$$

The total phase shift from the start of each wiggler segment to the start of the next segment is given by the sum of these three phase shifts [Eqs. (1-3)].

It is the total phase shift that is important for the detuning, which will be constant with respect to a phase shift in the gap (due, for example, by a change in the gap length) if the phase shift in the wiggler segment changes by a corresponding amount. The phase shift in the wiggler segment depends upon the wavelength that is excited, and is given by one wavelength per wiggler period only on resonance. Thus, a change in the gap length by $\delta L_{\text {gap }}$ will result in a change in the wavelength of

$$
\delta \lambda=-\frac{\delta L_{\mathrm{gap}}}{L_{w}} \frac{\lambda_{\mathrm{yes}}}{1+K_{\mathrm{rms}}^{2}}=-\frac{\delta L_{\mathrm{gap}}}{2 \gamma_{b}^{2} N_{w}},
$$

where $\gamma_{b}$ is the relativistic factor of the beam and $N_{w}$ (= $\left.L_{w} / \lambda_{w}\right)$ is the number of periods in the wiggler segment. As a consequence, introducing the same phase shift in each of the gaps will effectively retune the wavelength that is excited [21]. In order to suppress the fundamental, therefore, a variety of different phase shifts (i.e., gap lengths) must be imposed between different wiggler segments [13].

While we consider tuning the interaction by changing the gap lengths between the undulators, it is clear this is impractical in an operating x-ray FEL. However, as mentioned previously, it is also possible to vary the phase shift between the undulators by inserting a mild chicane in the gaps. This would have the advantage that it would allow the phase shift to be varied by the simple expedient of adjusting the current in the dipoles.

\section{SIMULATION RESULTS}

The electron beam, wiggler, radiation, and quadrupole parameters are summarized in Table I. We consider a beam with a kinetic energy of $12 \mathrm{GeV}$, which corresponds to a 
TABLE I. Simulation parameters.

\begin{tabular}{lc}
\hline \hline Electron beam & \\
\hline Energy & $12 \mathrm{GeV}$ \\
Peak current & $3400 \mathrm{~A}$ \\
Normalized emittance & $0.2 \mathrm{~mm}-\mathrm{mrad}$ \\
Energy spread & $0.01 \%$ \\
$\beta$ function & $8-40 \mathrm{~m}$ \\
Wiggler & Flat pole face \\
Period & $2.49 \mathrm{~cm}$ \\
Magnitude & $10.5134 \mathrm{kG}$ \\
$K_{\text {rms }}$ & 1.73 \\
Length & $2.5647 \mathrm{~m}$ \\
Radiation & \\
Fundamental wavelength & $0.9 \AA$ \\
Quadrupoles & \\
Field gradient & $3-19 \mathrm{kG} / \mathrm{cm}$ \\
Length & $0.0744 \mathrm{~m}$ \\
\hline \hline
\end{tabular}

fundamental resonance at a wavelength of $0.9 \AA$ for a wiggler with a period of $2.49 \mathrm{~cm}$ and an on-axis amplitude of $10.5134 \mathrm{kG}$. The peak current of the beam is assumed to be $3400 \mathrm{~A}$ and the normalized emittance and rms energy spread are $0.2 \mathrm{~mm}-\mathrm{mrad}$ and $0.01 \%$ respectively. Note that each wiggler segment is composed of 103 periods having uniform field strengths, but since MEDUSA simulates the entry and exit of the beam, two additional periods are included to model the entry and exit tapers. We inject matched beams as the $\beta$ function is changed and this requires injecting the beam with different Twiss parameters (in this case, the rms beam size and Twiss- $\alpha$ parameters) and also changing the field gradients in the quadrupoles. Since the wigglers focus in one plane, this means that the beam sizes in the $x$ and $y$ directions and, hence, $\beta_{x}$ and $\beta_{y}$, may be different. In practice, we have empirically determined $\beta_{x}$ and $\beta_{y}$ using MEDUSA, and that the values appearing in the various cases represent solutions that give near-optimal focusing. It is important to study the variation in the emission with $\beta$ function since the optimal $\beta$ functions differ for the fundamental and harmonics [13].

In this paper, we perform steady-state (i.e., single-slice) simulations to study the effect of detuning on the generation of both the fundamental and third harmonic. We first consider the effect of tuning the cavity length for $\beta_{x}=8.09 \mathrm{~m}$ and $\beta_{y}=3.82 \mathrm{~m}$ and plot the saturated power at the fundamental (blue) and third harmonic (red) in Fig. 2 as determined by MEDUSA simulations as functions of the gap length, $L_{\text {gap }}$, and the normalized gap length, $L_{\text {gap }} /\left[\lambda_{w}\left(1+K_{\mathrm{rms}}^{2}\right)\right]$. This value of $\beta$ corresponds to the optimal performance of the fundamental. Note that a change in the normalized gap length of unity corresponds to a phase shift of $2 \pi$. It is evident that there are peaks in the harmonic emission corresponding to phase shifts of approximately every $2 \pi / 3$ as indicated by intervals in the normalized gap length of $1 / 3$. These peaks in the

$$
L_{\text {gap }}(\mathrm{m})
$$

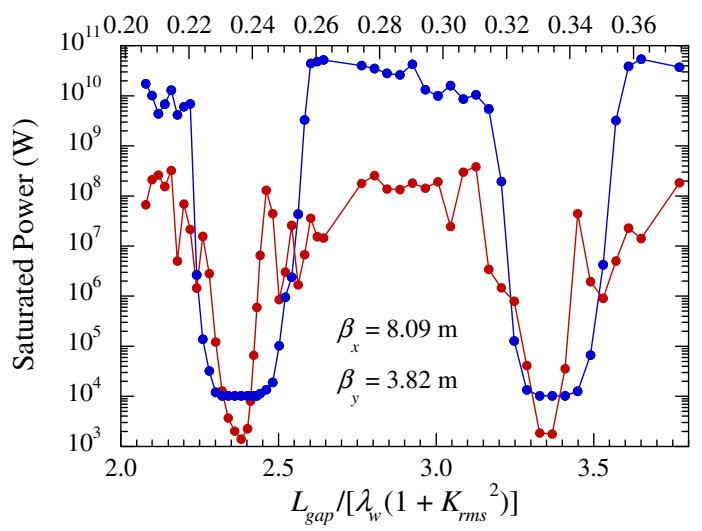

FIG. 2 (color online). Variation in the saturated power for the fundamental (blue) and third harmonic (red) for $\beta ; x=8.09 \mathrm{~m}$ and $\beta ; y=3.82 \mathrm{~m}$ as simulated by MEDUSA.

harmonic emission correspond to NHG when the fundamental is not detuned and to LHG when the fundamental is detuned. In either case, however, the peaks in the harmonic emission reach about the same power levels. These harmonic peaks are reduced relative to the fundamental by about 2-3 orders of magnitude, which is what is typically expected of the harmonic emission. However, this choice of the $\beta$ functions is near the optimal values for fundamental emission. As will be discussed, the harmonic emission is substantially enhanced for increased values of the $\beta$ functions.

The optimal gap length for fundamental emission, as determined by the minimum gain length, occurs for $L_{\text {gap }} /\left[\lambda_{w}\left(1+K_{\text {rms }}^{2}\right)\right] \approx 2.6228\left(L_{\text {gap }} \approx 0.26040 \mathrm{~m}\right)$ over the range of gap lengths shown. At this gap length, the NHG mechanism is dominant, and the evolution of the fundamental and third harmonic are shown in Fig. 3. The evolution of the harmonic exhibits the expected behavior of the NHG mechanism; specifically, there is linear harmonic growth following the initial start-up region followed by nonlinear harmonic growth once the fundamental reaches

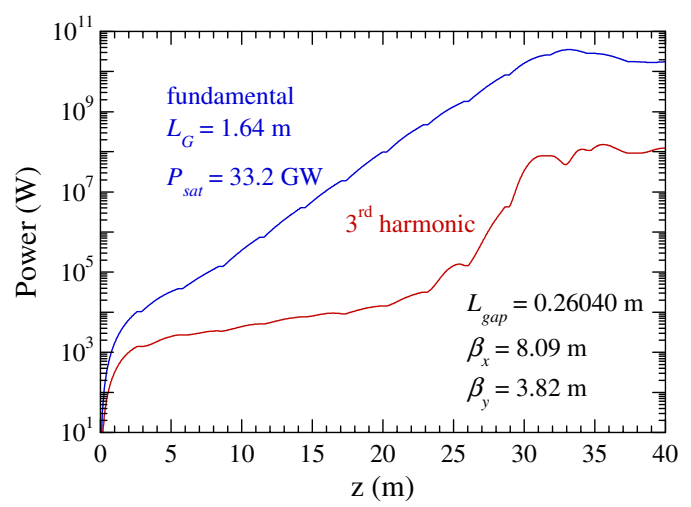

FIG. 3 (color online). Evolution of the fundamental and third harmonic for $L_{\text {gap }}=0.2604 \mathrm{~m}$. 


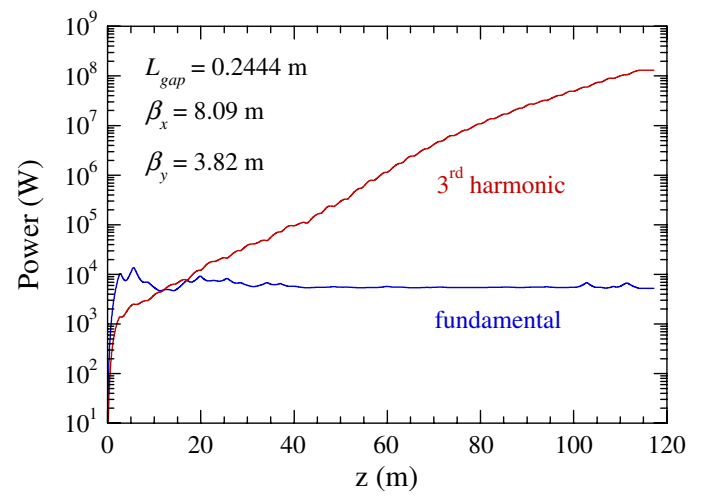

FIG. 4 (color online). Evolution of the third harmonic for $L_{\text {gap }}=0.2444 \mathrm{~m}$.

high powers. For this case, LHG is observed over the range of about $5 \mathrm{~m}<z<26 \mathrm{~m}$ after which NHG is dominant. Saturation of the third harmonic is found slightly before the fundamental saturates at a distance of about $34 \mathrm{~m}$. Note that since the phase shift is periodic, this performance for the fundamental will be replicated for integer increments of this normalized phase shift.

The third harmonic is found to grow exponentially for a gap length where the fundamental is suppressed. This is LHG and is shown in Fig. 4 where we plot the evolution of the third harmonic power as a function of position along the wiggler line. For comparison, we also plot the evolution of the fundamental. Observe that the saturation distance is now over $100 \mathrm{~m}$, which results from the longer gain length for LHG of the harmonic.

As previously mentioned, the choice of $\beta_{x}=8.09 \mathrm{~m}$ and $\beta_{y}=3.82 \mathrm{~m}$ is near the optimal values for performance at the fundamental, and this is in good agreement with predictions based on the parametric scaling developed by Ming Xie [22]. A comparison of the fundamental gain length (blue circles) and saturated power found in simulation (red triangles) and the parametrization due to Ming Xie (blue and red lines) is shown in Fig. 5 versus $\beta_{x}$.

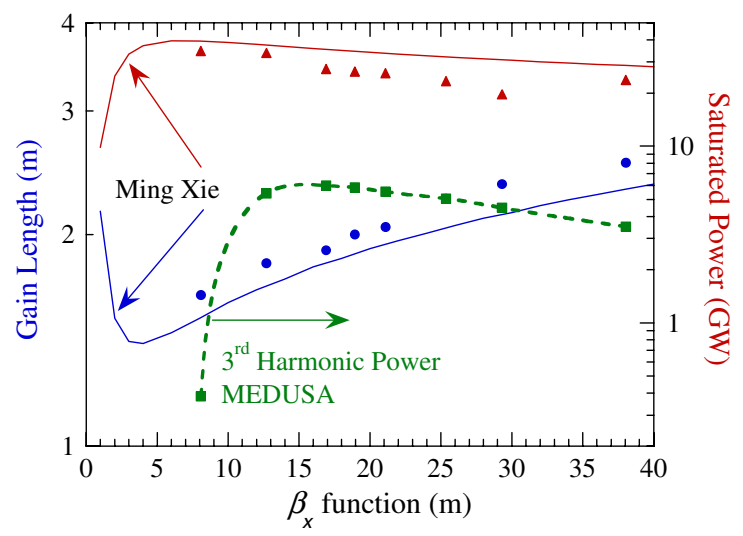

FIG. 5 (color online). Comparison of the simulation with MEDUSA and the parametrization due to Ming Xie [18].
Also shown in Fig. 5 is the maximum saturated power of the third harmonic as found in simulation (green squares), where the dashed line connecting the points is simply a fit to the data and not an analytic extension of the Ming Xie parametrization. Note that the peak powers as found with NHG and LHG are comparable. Observe that the qualitative behavior of the third harmonic is similar to that of the fundamental in that there is a relatively rapid increase in power as $\beta$ increases up to the optimal value of $\beta$, followed by a relatively slow decline thereafter. For the third harmonic, the optimal value for $\beta$ is approximately $18 \mathrm{~m}$ for these beam, wiggler, and quadrupole parameters. There is a substantial enhancement in harmonic power with respect to that found at the $\beta$ that yields the maximum power at the fundamental. The third harmonic power when $\beta_{x}=8.09 \mathrm{~m}$, corresponding to the optimal fundamental power, is about $380 \mathrm{MW}$, but rises to about $6.0 \mathrm{GW}$ when $\beta_{x}=18 \mathrm{~m}$. This is an impressive increase of more than an order of magnitude in the third harmonic power.

The variation of the saturated power at the fundamental (blue) and the third harmonic (red) as functions of gap length found in simulation for the near-optimal $\beta$ for the third harmonic is shown in Fig. 6. Observe that the periodicities indicated in Fig. 2 for smaller values of $\beta$, are more pronounced here where the third harmonic is more prominent.

The simulations showing the variation in the tuning with gap length were done at a fixed wavelength. However, since the phase shift from the start of one wiggler to the next varies substantially with wavelength over the amplification band, changes in the gap will result in changes in the wavelength excited within the resonant bandwidth. This is illustrated for the fundamental in Fig. 7, which is a contour plot of the fundamental output power versus both wavelength and (normalized) gap length. Observe that the peak powers are found for wavelengths close to $0.9( \pm 0.004) \AA$ and that the output is periodic in gap length.

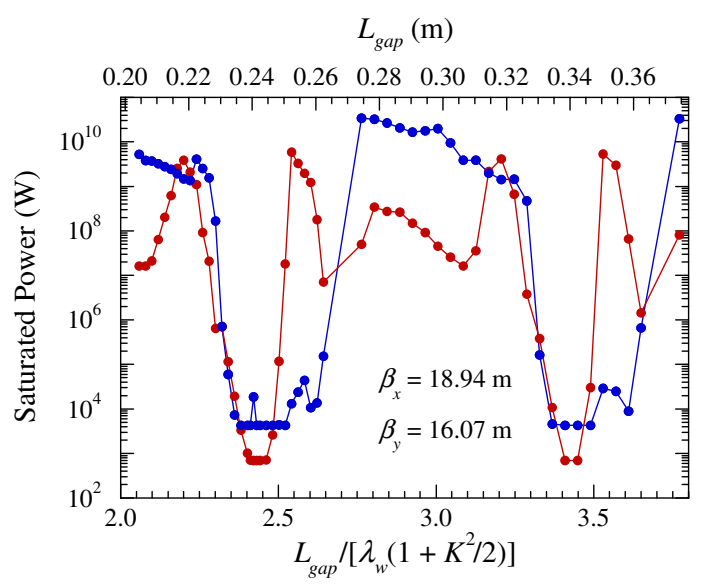

FIG. 6 (color online). Variation in the saturated power at the fundamental and third harmonic for the optimal $\beta$; for third harmonic generation. 


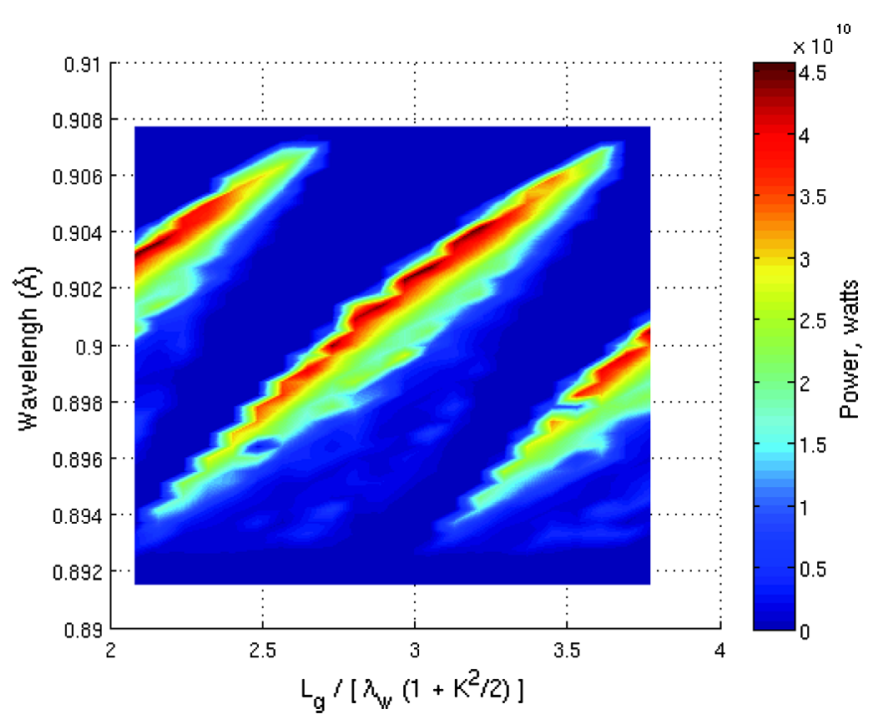

FIG. 7 (color online). Contour plot showing the variation in fundamental power with the wavelength and normalized gap length.

A corresponding contour plot of the third harmonic power versus both wavelength and (normalized) gap length is shown in Fig. 8. In this case, we note that there are 5 peaks corresponding to the NHG and LHG harmonic peaks over the range shown in Figs. 2 and 8, although the central peak is much reduced relative to the outer peaks.

Figures 7 and 8 provide a measure of the bandwidth that would be excited at any given gap length, as well as the relative magnitude of the radiated powers.

It is interesting to compare the performance of the third harmonic at a wavelength of $0.3 \AA$ for a wiggler tuned to a fundamental wavelength of $0.9 \AA$ as described previously

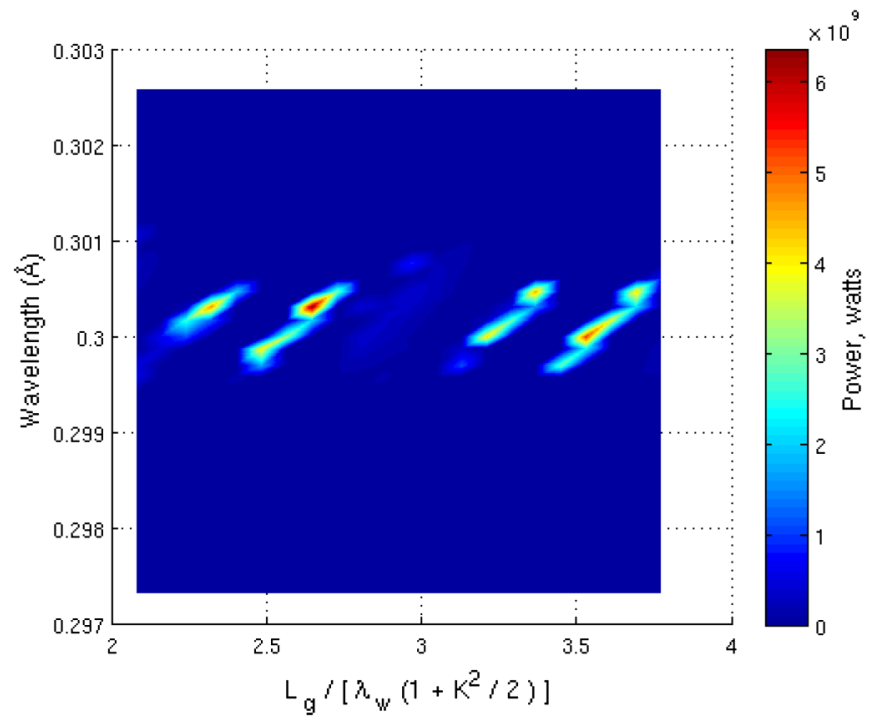

FIG. 8 (color online). Contour plot showing the variation in the third harmonic power with wavelength and normalized gap length.

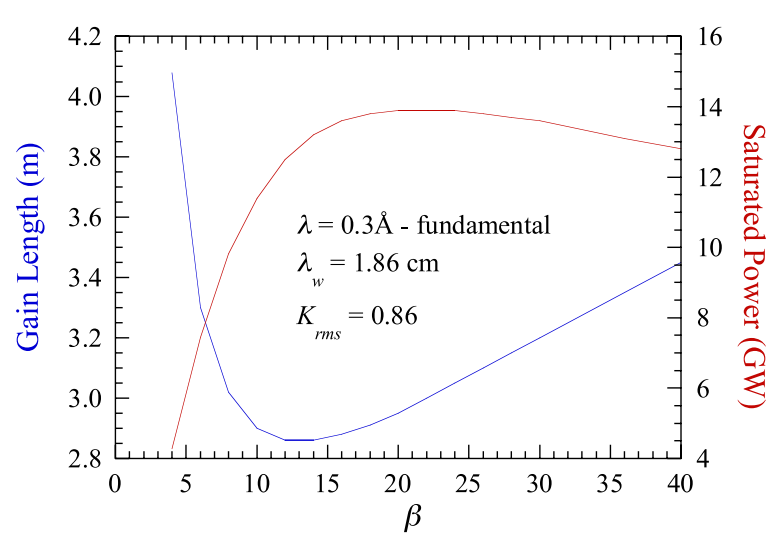

FIG. 9 (color online). Gain length and saturation power for wiggler parameters consistent with operation at a fundamental wavelength of $0.3 \AA$ found using the parametrization due to Ming Xie [22].

with the performance at $0.3 \AA$ when the wiggler is tuned to a fundamental at that wavelength. To tune the FEL to lase at $0.3 \AA$ at the fundamental we use the same beam parameters as shown in Table I but we reduce the wiggler period and amplitude. In particular, when tuned to the fundamental, we use a wiggler period of $1.86 \mathrm{~cm}$ and a peak on-axis amplitude of $7.0 \mathrm{kG}$ yielding an rms wiggler strength parameter of $K_{\text {rms }}=0.86$. It should be remarked that a wiggler producing this field would have the same gap as the field used in the harmonic simulations. As shown previously in Fig. 5, the performance at the fundamental found in simulation with MEDUSA is in close agreement with the results of the parametrization developed by Ming Xie [22]. In view of this, we show the predicted performance at the fundamental of $0.3 \AA$ using the parametrization in Fig. 9 where we plot the gain length (blue) and the saturated power (red) versus $\beta$. As shown in the figure, the saturated power at the fundamental $(0.3 \AA)$ reaches a maximum value of about $14 \mathrm{GW}$ when $\beta=20 \mathrm{~m}$. This is only a factor of about 2.3 greater than the highest power found when the wiggler is tuned to a third harmonic at $0.3 \AA$ and $\beta=18 \mathrm{~m}$. In a more general sense, although we have not included such simulations herein, we speculate that it is possible to achieve nearly the same power levels at a fixed wavelength using a harmonic interaction at either a lower energy or a longer wiggler period than comparable operation at the fundamental.

\section{SUMMARY AND DISCUSSION}

In this paper, we analyzed enhanced harmonic generation in $\mathrm{x}$-ray FELs. Following an analysis by Schneidmiller and Yurkov [13] that harmonic generation can be enhanced by proper tuning of the $\beta$ function, we performed three-dimensional simulations using the MEDUSA simulation code of an X-ray FEL design based upon a FODO lattice composed of multiple wiggler 
segments in which quadrupoles are inserted in the gaps between the wigglers.

Steady-state simulations were performed where the fundamental $(0.9 \AA)$ and third harmonic $(0.3 \AA)$ powers were obtained as functions of both the gap length between the wigglers and the $\beta$ function of the FODO lattice. As discussed in [13], it was found that the optimal $\beta$ for fundamental generation differed from that for the third harmonic. For the case under consideration, the third harmonic power for the choice of $\beta(\approx 8 \mathrm{~m})$ that resulted in the maximum fundamental power was about $1 \%$ that of the fundamental. However, the harmonic power increased rapidly with $\beta$ until it reached a peak for $\beta \approx 17 \mathrm{~m}$ where the third harmonic power reached about $22 \%$ of the fundamental power (at this $\beta$ ). Further, comparison of the optimal 3rd harmonic power at $0.3 \AA$ compared favorably with the predicted performance for a wiggler tuned to a fundamental at $0.3 \AA$.

It should be remarked that, as indicated in Figs. 7 and 8, variations in the gap length will result in a shift in the excited wavelengths of the fundamental and harmonics. Because of this, time-dependent simulations, which include the full spectrum of excited waves, can be expected to show a shift in the excited spectrum rather than a complete detuning of the fundamental. It was for this reason that Schneidmiller and Yurkov [13] included varying the gap lengths between the different wiggler segments in order to detune the fundamental. A more complete analysis using time-dependent simulations is in progress; however, the complete detuning of the fundamental may be a difficult process. Nevertheless, the NHG mechanism requires strong growth of the fundamental, and will be significantly enhanced by the proper tuning of the FODO lattice to optimize the $\beta$ function for harmonic generation.

In summary, we conclude that harmonic generation can be substantially enhanced by the optimal choice of $\beta$ for the FODO lattice in an $x$-ray FEL. In addition to the work described here, we (1) expect that the harmonic generation can be enhanced still further by the use of a tapered wiggler, and (2) speculate that different harmonics may be preferentially enhanced by different choices of $\beta$ for the FODO lattice.

\section{ACKNOWLEDGMENTS}

We gratefully acknowledge the support of the U.S. Department of Energy through the LANL/LDRD
Program for this work. This research used resources provided by the Los Alamos National Laboratory Institutional Computing Program, which is supported by the U.S. Department of Energy National Nuclear Security Administration under Contract No. DE-AC5206NA25396.

[1] H. P. Freund and T. M. Antonsen, Jr., Principles of FreeElectron Lasers (Chapman \& Hall, London, 1996), 2nd ed.

[2] P. Emma et al., Nat. Photonics 4, 641 (2010).

[3] T. Shintake, Nat. Photonics 6, 540 (2012).

[4] R. Bonifacio, L. De Salvo, and P. Pierini, Nucl. Instrum. Methods Phys. Res., Sect. A 293, 627 (1990).

[5] H. P. Freund, S. G. Biedron, and S. V. Milton, IEEE J. Quantum Electron. 36, 275 (2000).

[6] Z. Huang and K.-J. Kim, Phys. Rev. E 62, 7295 (2000).

[7] E. L. Saldin, E. A. Schneidmiller, and M. V. Yurkov, Phys. Rev. ST Accel. Beams 9, 030702 (2006).

[8] P. E. Latham, B. Levush, T. M. Antonsen, Jr., and N. Metzler, Phys. Rev. Lett. 66, 1442 (1991).

[9] L. Giannessi and P. Musumeci, New J. Phys. 8, 294 (2006).

[10] H. Deng, H. Bei, and Z. Dai, Chin. Phys. C 34, 115 (2010).

[11] J. Dai, H. Deng, and Z. Dai, Phys. Rev. Lett. 108, 034802 (2012).

[12] B. W. J. McNeil, G. R. M. Robb, M. W. Poole, and N. R. Thompson, Phys. Rev. Lett. 96, 084801 (2006).

[13] E. A. Schneidmiller and M. V. Yurkov, Phys. Rev. ST Accel. Beams 15080702 (2012).

[14] H. P. Freund, Phys. Rev. A 27, 1977 (1983).

[15] H. P. Freund, Phys. Rev. ST Accel. Beams 8, 110701 (2005).

[16] H. P. Freund, L. Giannessi, and W. H. Miner, Jr., J. Appl. Phys. 104, 123114 (2008).

[17] X. J. Wang, T. Watanabe, Y. Shen, R. K. Li, J. B. Murphy, T. Tsang, and H. P. Freund, Appl. Phys. Lett. 91, 181115 (2007).

[18] X. Wang, H. Freund, D. Harder, W. Miner, J. Murphy, H. Qian, Y. Shen, and X. Yang, Phys. Rev. Lett. 103, 154801 (2009).

[19] P. van der Slot, H. Freund, W. Miner, Jr., S. Benson, M. Shinn, and K.-J. Boller, Phys. Rev. Lett. 102, 244802 (2009).

[20] L. Giannessi et al., Phys. Rev. ST Accel. Beams 14, 060712 (2011).

[21] H. P. Freund, Phys. Rev. E 70, 015501(R) (2004).

[22] M. Xie, Proceedings of the Particle Accelerator Conference, Dallas, TX, 1995 (IEEE, New York, 1995). 